\title{
Ultrasound-Augmented Phase Transition Nanobubbles for Targeted Treatment of Paclitaxel-Resistant Cancer
}

Yi Zhu ${ }^{\mathrm{a}, \mathrm{b}, 1}$, Guonan Zhang ${ }^{\mathrm{c}, 1}$, Meiying Li ${ }^{\mathrm{d}}$, Lang Ma ${ }^{\mathrm{a}}$, Jianming Huang ${ }^{\mathrm{d}, *}$, Li Qiu ${ }^{\mathrm{a}, *}$

${ }^{a}$ Department of Ultrasound, West China Hospital, Sichuan University, Chengdu 610041, China.

b Department of Ultrasound, the Affiliated Cancer Hospital, School of Medicine, University of Electronic Science and Technology of China, Sichuan Cancer Hospital \& Institute, Chengdu 610041, China.

c Department of Gynecological Oncology, the Affiliated Cancer Hospital, School of Medicine, University of Electronic Science and Technology of China, Sichuan Cancer Hospital \& Institute, Chengdu 610041, China.

d Department of Biochemistry \& Molecular Biology, the Affiliated Cancer Hospital, School of Medicine, University of Electronic Science and Technology of China, Sichuan Cancer Hospital \& Institute, Chengdu 610041, China.

${ }^{1}$ Y.Z. and G.N.Z. contributed equally to this work.

\section{*Corresponding Authors:}

E-mail: qiulihx@scu.edu.cn or wsqiuli@126.com (L. Qiu), wesleyhuangcn2002@163.com (J. M. Huang). 

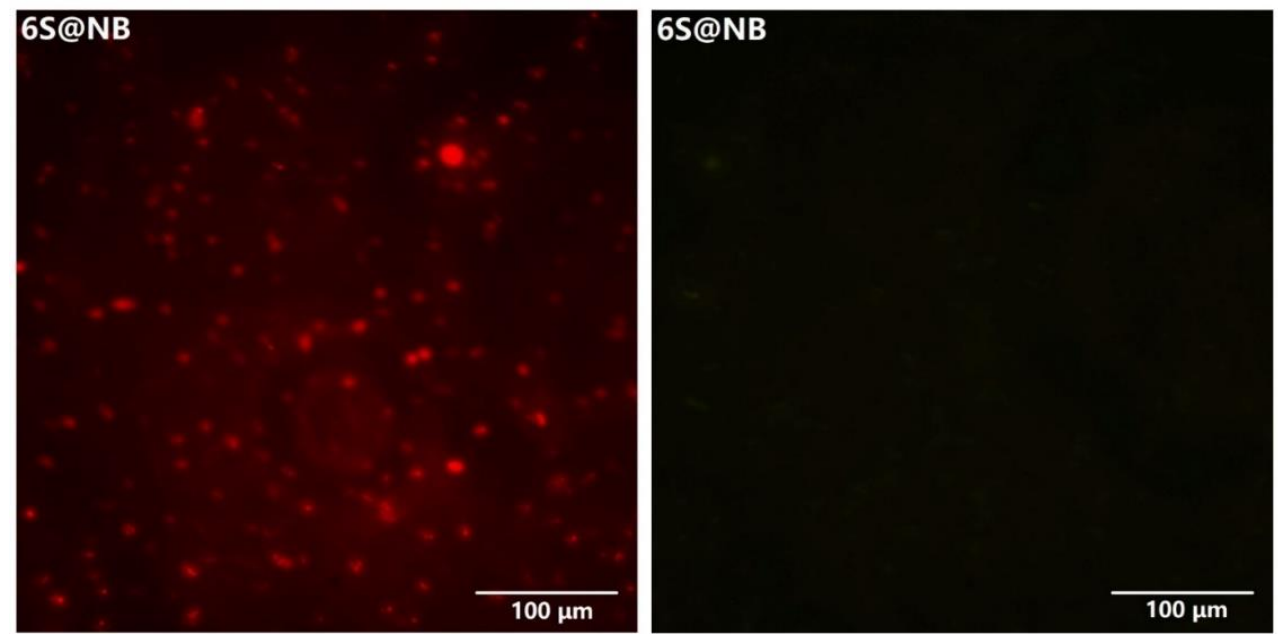

Fig. S1 Dil-stained unconjugated 6S@NB control showed (left) red without (right) green (SABAlexa488) fluorescence.

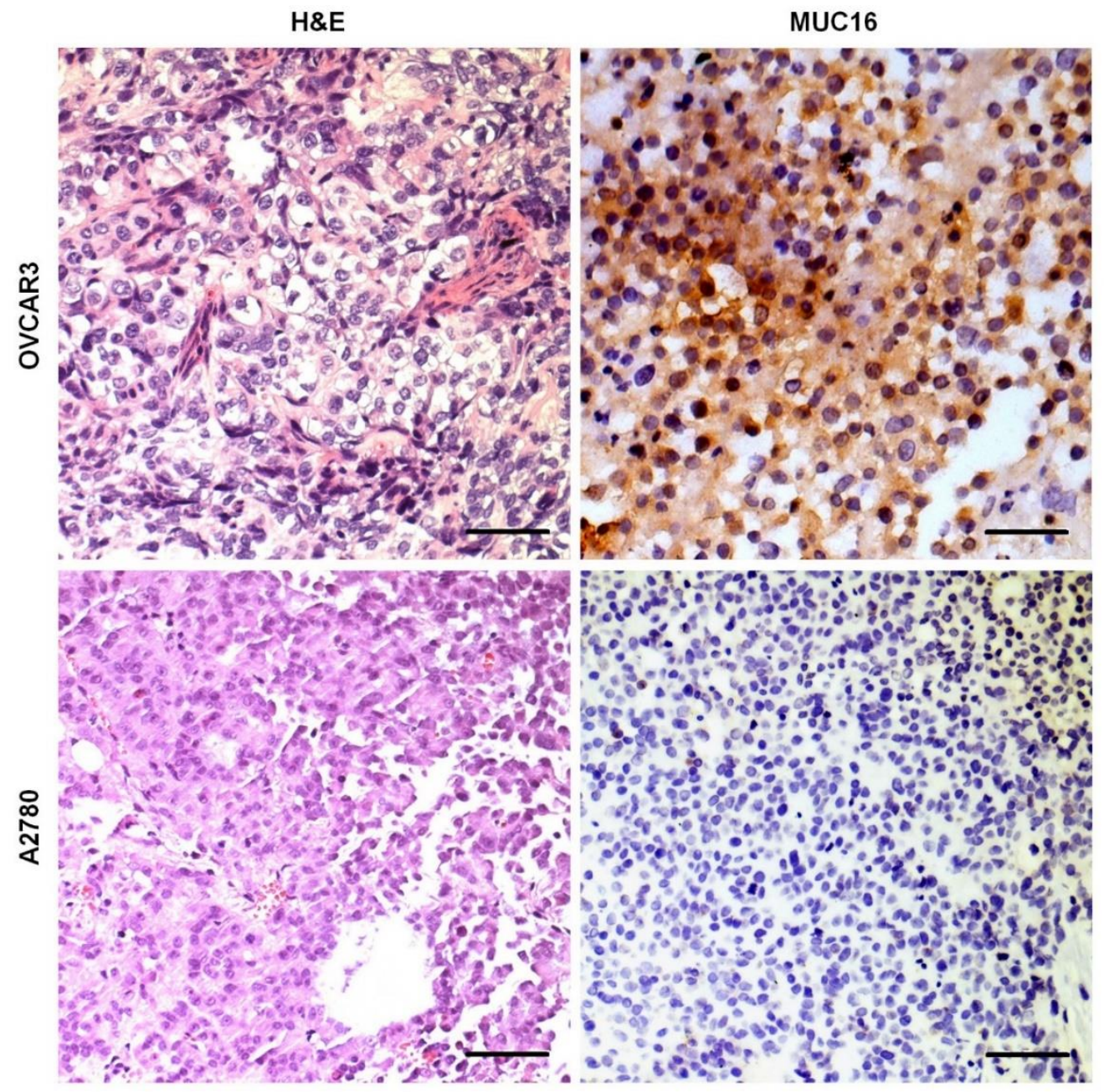

Fig. S2 Expression of MUC16 in OVCAR3 and A2780 derived tumors in tumor xenograft models (The scale bar is $50 \mu \mathrm{m}$ ). In contrast to the situation in A2780 tumor tissue, strong immunostaining of MUC16, which was localized in the membrane and cytoplasm, was observed in OVCAR3 tumor tissue. 